\title{
Im Diskurs bestehen. Über den notwendigen Pluralismus in der ökonomischen Politikberatung: Replik zum Kommentar von Stefan Kooths
}

\author{
Sebastian Dullien • Gustav Horn
}

Online publiziert: 22. Februar 2019

(C) List-Gesellschaft e.V. 2019

Stefan Kooths hat einen sehr aufschlussreichen und damit interessanten Kommentar zu unserem Text geliefert. Kooths beginnt völlig richtig mit der Feststellung, dass es „,der Debatte nicht gut [tut], die Gegenseite in die Nähe anderslautender Positionen zu rücken“. Bedauerlicherweise können wir uns bei Lektüre seines Kommentars nicht des Eindrucks erwehren, dass er genau dies mit seinem Text getan hat.

So behauptet Kooths etwa, dass wir den Vertretern der ,unreformierten Orthodoxie“ ein „fundamentales Unverständnis der wissenschafts- und erkenntnistheoretischen Grundlagen“ vorgeworfen hätten. Davon ist in unserem Text nirgendwo die Rede. Mal abgesehen davon, dass fraglich ist, wer sich überhaupt als ,unreformierter Orthodoxer" angesprochen fühlen würde (wir selber bezeichnen niemanden mit diesem Label, sondern benutzen den Begriff, um den Eindruck anderer wiederzugeben), geht unser Vorwurf des Unverständnisses der wissenschaftstheoretischen Grundlagen nicht an den Mainstream der deutschen Ökonomen, sondern an jene Untergruppe, die Pluralismus aus den wirtschaftspolitischen Beratungsinstitutionen heraushalten will. Dies ist ein enormer Unterschied. Ein beachtlicher Teil des Mainstreams, mit dem wir in der ein oder anderen methodischen oder inhaltlichen Frage nicht übereinstimmen, würde kaum die Forderung nach mehr Homogenität in der Politikberatung unterschreiben.

This reply refers to the commentary article available online at https://doi.org/10.1007/s41025-01900150-7.

\footnotetext{
S. Dullien $(\bowtie)$

Hochschule für Technik und Wirtschaft, Berlin, Deutschland

E-Mail: Sebastian.Dullien@HTW-Berlin.de

G. Horn

Institut für Makroökonomie und Konjunkturforschung, Düsseldorf, Deutschland

E-Mail: gustav-horn@boeckler.de
} 
Kooths scheint außerdem zu implizieren, dass wir die Position vertreten haben, dass sich unterschiedliche Paradigmen durch unterschiedliche zugrunde liegende Werturteile definieren, wenn er schreibt, dass sich ein Paradigma ,nicht durch Werturteile im Sinne politischer Ziele auszeichnet" (3. Absatz). Dabei haben wir so etwas nicht behauptet und würden es auch nicht tun. Paradigmen sind Annahmen darüber, wie etwas (in unserem Fall die Wirtschaft) funktioniert und welche Kanäle relevant oder weniger relevant sind.

In Bezug auf Paradigmen und Werturteile haben wir einzig die empirische Beobachtung gemacht, dass Werturteile damit korreliert sind, zu welchen Schlussfolgerung Ökonomen bei Vorliegen der gleichen Faktenlage kommen (selbst wenn sie an den gleichen Universitäten promoviert haben und man deshalb von einem gleichen Qualifikationsniveau ausgehen kann) ${ }^{1}$. Wenn die Annahmen über die Funktionsmechanismen der Wirtschaft - wie oben - als Paradigma definiert sind, so folgt daraus, dass Werturteile von Ökonomen mit den Paradigmen korreliert sind, welchen sie folgen.

Da dies ein Phänomen ist, dass die Ökonomie von Naturwissenschaften unterscheidet und die Kausalität zwischen Werten und Paradigma in beide Richtungen laufen kann, sind Politiker gut beraten, bestimmte Ratschläge von Ökonomen mit großer Vorsicht zu betrachten, da diese möglicherweise implizite Werturteile enthalten, die der Politiker möglicherweise nicht teilt. Und wenn Politikern Ratschläge nur aus einer einzelnen paradigmatischen Richtung vorgesetzt werden, besteht die Gefahr, dass die Politiker die Ratschläge als durch wertbeladende Vorurteile verzerrt ansehen, und ökonomischen Rat am Ende gänzlich ablehnen, obwohl auch Ökonomen mit anderen paradigmatischen Ansätzen zu einem ähnlichen Ergebnis gekommen wären.

Wir sind auch der Meinung, dass wir in unserem Text hinreichend klar gemacht haben, dass wir Pluralität nicht ,,als Nebeneinander verschiedener Denkschulen“ und nicht als „Ersatz für ein mühsames, nach den Kriterien der Wissenschaft geführtes Ringen um Erkenntnisfortschritt" sehen, wie uns Kooths unterstellt. Vielmehr haben wir - gerade auch mit der Forderung, dass die Politik als Auftraggeber von Expertisen die beteiligten Wissenschaftler zum Dialog zwischen den Paradigmen verpflichten sollte - gerade ein solches Ringen um den Erkenntnisfortschritt eingefordert. Wir haben es in Anlehnung an Hellwig sogar als vornehmste Aufgabe einer wissenschaftlichen Politikberatung definiert, Bedingtheit und Reichweite der Annahmen vieler Modelle mit wissenschaftlichen Methoden zu untersuchen.

Kooths behauptet weiter, wir würden ,suggerieren, dass die Wahrheit bei widerstreitenden Paradigmen irgendwo in der Mitte liegt“. Wir haben so etwas nicht geschrieben, und es fällt uns auch schwer, für diese „Suggestion“ Anhaltspunkte in unserem Text zu finden. Uns ist klar, dass bei Fragen etwa der Wirkung nach Austerität auf Output und Beschäftigung nicht die Wahrheit zwischen der Idee der traditionell keynesianischen Multiplikatoren (die einen negativen Effekt prognostizieren) und der Idee nicht-keynesianischen Effekte (die einen positiven Effekt sehen

\footnotetext{
${ }^{1}$ Hier sei noch einmal auf die von uns vorne bereits zitierten Arbeiten von Fuchs et al. (1998), Gordon und Dahl (2013) und May et al. (2018) verwiesen.
} 
würden) nicht bei einem Durchschnittseffekt von null liegt, und wir dachten eigentlich, dies in unserem Text hinreichend klargestellt zu haben.

Unser Punkt ist, dass jedes Paradigma fehlbar ist, und dass gerade am Rand des wissenschaftlichen Erkenntnisstands, bei der Analyse wichtiger aktueller wirtschaftspolitischer Themen (wie etwa dem gesetzlichen Mindestlohn, der Konjunkturpolitik, und, natürlich auch der - von Ökonomen in der österreichischen Tradition gestellten Frage des Zusammenhangs von Geldpolitik und Vermögenspreisblasen) die Unsicherheit besonders groß ist.

Kooths argumentiert, dass das Auswählen zwischen den richtigen Antworten bei umstrittenen Fragen eine Aufgabe der Wissenschaft sei und nicht der Politik überlassen werden dürfe, weil diese mangels inhaltlicher Sachkenntnis nicht in der Lage seien, die Güte verschiedener Ansätze gegeneinander abzuwägen. Kooths hat dabei Recht, dass die Politik am Ende nicht über die wissenschaftliche Qualität oder empirische Treffsicherheit der Ansätze entscheiden kann. Darum geht es aber auch gar nicht. Der Politik soll die Analyse aus unterschiedlichen Blickwinkeln vorgelegt werden, damit sie erkennen kann, mit welchem Grad der Sicherheit eine wissenschaftliche Erkenntnis behaftet ist. So dürften praktisch alle Paradigmen zu dem Schluss kommen, dass eine Einführung einer deutlichen Steuer auf „Alcopops“ (alkoholischer Mischgetränke aus Limonade und harten Alkoholika) einen massiven Rückgang der nachgefragten Menge zum Ergebnis hat. In diesem Fall würden alle Gutachten aus unterschiedlichen paradigmatischen Richtungen die gleiche Antwort geben, und der Politik wäre klar, dass die Antwort dieser Frage mit großer Sicherheit so beantwortet werden kann. Bei anderen Fragen (wie etwa der Wirkung von Fiskalpolitik oder Austerität oder eines gesetzlichen Mindestlohns) würden die Ergebnisse kontroverser ausfallen, und die Politik wüsste, dass sie diese größere Erkenntnisunsicherheit in ihre Entscheidung einfließen lassen muss.

Das Aufzeichnen von Unsicherheiten ist für die Politik zentral, weil üblicherweise alle Handlungsoptionen mit Kosten und Nutzen versehen sind. Um bei Kooths Beispiel eines Autos zu bleiben, dass auf eine Mauer zurast. Sind die Bremsen defekt, bleibt dem Autofahrer als Handlungsoption, rechts oder links von der Straße zu fahren oder eben die Mauer zu treffen. Befindet sich neben der Straße ein Abhang oder Bäume, so bleiben die Alternativen eines Zusammenstoßes mit der Mauer, mit einem Baum oder das Überschlagen am Abhang. Um diese Entscheidung richtig fällen zu können, ist es nun sehr wohl wichtig zu wissen, mit welcher Wahrscheinlichkeit die Mauer aus Stein oder Styropor besteht, oder ob es sich gar um eine Fata Morgana handelt. Sind alle Experten der Meinung, es handele sich um eine massive Steinmauer, könnte die Entscheidung anders ausfallen, als wenn nur $51 \%$ der Wissenschaftler dieser Überzeugung sind, die anderen $49 \%$ aber die Mauer als eine aus Styropor oder Luft sehen.

Dieses Aufzeigen der Grenzen und Unsicherheit der wissenschaftlichen Erkenntnisse wird eine monoparadigmatische Politikberatung nie wirklich leisten können. Wissenschaftler haben sich für ein Paradigma entschieden, weil sie dieses für besser als die anderen Paradigmen halten. Da auch die Psychologie immer wieder aufzeigt, dass menschliche Individuen kaum in der Lage sind, eigene Vorurteile als solche zu erkennen, kann man nicht erwarten, dass Wissenschaftler objektiv die Unsicherheit der eigenen Ansätze darstellen können oder werden. Ein echtes Aufzeigen der 
Unsicherheiten kann nur über multiparadigmatischen Dialog erreicht werden. Eine Lektüre der Gutachten des Sachverständigenrats aus den vergangenen Jahren bestätigt diesen Eindruck: Jenseits der Minderheitsvoten findet man dort - auch bei wissenschaftlich umstrittenen Fragen - selten ein klares Eingeständnis der großen Unsicherheiten, mit denen die Empfehlungen des Rates in diesen Feldern naturgemäß behaftet sind.

Kooths selber schreibt dazu: „Wahrheit ist [...] keine Frage der politischen Mehrheit." Hier können wir nur zustimmen. Allerdings zeigt die Wissenschaftsgeschichte, dass Wahrheit eben auch keine Frage der Mehrheit innerhalb der Profession ist.

Wie Kooths zu Recht schreibt, ist es eigentlich Aufgabe der Wissenschaft, den Streit zwischen unterschiedlichen Paragdimen auszufechten. Das Problem ist nur, dass die Wissenschaft dieser Aufgabe gelegentlich nachkommt, in anderen Fällen aber massiv versagt, und dass es - wie Solomon (2007) ausführt, keinen empirischen oder theoretischen Grund gibt, anzunehmen, dass die Ergebnisse des Wissenschaftssystems hier effizient wären. Wie Hans-Jürgen Krupp (2018) mit weiteren Beispielen aus der deutschen Hochschullandschaft und Politikberatung illustriert, kann nicht davon ausgegangen werden, dass die Wissenschaft von sich aus die Forschung oder die Vielfalt, die Politikberatung braucht, bereit stellt. Die Politik als Nachfrager ist hier sehr wohl in der Lage, die Wissenschaft zu Dialog zwischen den Paradigmen zu zwingen, der sonst oft nicht stattfindet, da die Wissenschaft tatsächlich bestenfalls „permissiven Pluralismus“ praktiziert, bei denen andere Ansätze toleriert werden, eine echte Beschäftigung mit ihnen aber nicht stattfindet.

Auch das Argument, die Politik als Nachfrager könne nicht sinnvoll für Pluralität und Paradigmenvielfalt sorgen, weil ja neue, noch unbekannte Paradigmen per Definition nicht vom Staat nachgefragt werden könnten, ist bestenfalls auf den ersten Blick überzeugend: Der Anreiz zur Entwicklung neuer Paradigmen steigt mit dem Maße, dass sich Wissenschaftler Aufmerksamkeit für solche Paradigmen erhoffen können. Diese Aufmerksamkeit ist wesentlich wahrscheinlicher, wenn auch Paradigmen in der Politikberatung beachtet werden, die zwar eine gewisse Bekanntheitsschwelle erreicht, aber (noch) nicht den Status des Mainstreams erreicht haben. $\mathrm{Zu}$ behaupten, Pluralismus bestehender Paradigmen in der Politikberatung werde die Entwicklung neuer Alternativen in der Wissenschaft bremsen, wie es Kooths tut, ist absurd.

Es ist im Übrigen bestenfalls naiv, einerseits Politikern eine mikroökonomische Rationalität zu unterstellen, die nicht der wissenschaftlichen Wahrheitsfindung, sondern dem Sieg im politischen Kampf folgt, und andererseits anzunehmen, dass Wissenschaftler mikroökomisch allein vom Streben nach Erkenntnis geleitet seien. Man kann auch die Hypothese wagen, dass Politiker ein Interesse an richtigen ökonomischen Erkenntnissen haben, um Wahlerfolge zu erzielen, während Wissenschaftler von opportunistischem Statusdenken und der Sicherung von Ressourcen für die eigene Arbeit geprägt sind. Wir halten beide Hypothesen in ihrer Schlichtheit für falsch, aber damit auch die entsprechenden Aussagen von Kooths. Um seine Ablehnung von mehr Vielfalt und Pluralismus in der wirtschaftspolitischen Politikberatung zu unterstreichen, führt Kooths weiter als Holzhammer-Argument an, dass die Alternative zur homogenen Politikberatung ein ,,anything goes“ sei. Das Heranziehen des Begriffs ,,anything goes“ ist zwar in der Debatte um Pluralismus beliebt, ist aber leider 
erneut nichts anderes, als der Gegenseite eine absurde Position zu unterstellen, die man dann leicht angreifen kann. „Anything goes“ erweckt den Anschein, dass alles erlaubt sein solle, und Astrologie und religiöse Überzeugungen ebenso ein Anrecht auf Aufmerksamkeit hätten wie wissenschaftliche Ansätze. Darum geht es aber in der Pluralismus-Debatte nicht. Jenseits der Paradigmen gibt es ja einen (sozial-)wissenschaftlichen Konsens, was Wissenschaft ist und was nicht, wobei üblicherweise auf das systematische Aufstellung von Hypothesen und deren empirischer Überprüfung verwiesen wird. Und es gibt keinen Grund, Paradigmen, die diesen Prinzipien der Wissenschaftlichkeit folgen und empirische Erfolge vorweisen, von vorne herein auszuschließen.

$\mathrm{Zu}$ guter Letzt sind wir etwas verwundert, dass zwar Kooths eigentlich in der Politikberatung keine Notwendigkeit für Pluralismus ablehnt, dann aber eine Reihe von Fragen aus der österreichischen Schule aufwirft, die er stärker behandelt haben möchte, uns aber gleichzeitig vorwirft, durch die ,Wahl [der] Beispiele [...] nur auf keynesianische Erklärungsalternativen hingewiesen“ zu haben. Tatsächlich haben wir aus Platzgründen eigentlich nur ein Thema vertieft angesprochen - jenes zur Wirksamkeit von Fiskalpolitik zum einen im Rahmen von Austeritätsprogrammen, zum anderen als Konjunkturpolitik. Wir hatten eigentlich dieses Beispiel gewählt, weil nach unserer Lesart die Mainstream-Position zu dem Thema sich in relativ gut dokumentierter Weise in den vergangenen 15 Jahren verschoben hat. Kooths schreibt hier dagegen, dass die Position, diskretionäre Konjunkturpolitik habe zur Wirtschaftserholung nach 2008/9 beigetragen, sei weder ,im Nachhinein durch die Daten bestätigt oder gar als Konsens in den Mainstream eingegangen“. Darüber ließe sich trefflich streiten.

Nun ist die Interpretation von „Konsens im Mainstream“ gerade bei umstrittenen Themen schwierig. In einer Umfrage der Universität Chicago unter führenden Ökonomen äußerten allerdings 2014 mehr als 80\% der befragten hochrangigen Volkswirte (aus unterschiedlichen politischen Richtungen), das (diskretionäre) USStimulus-Paket American Recovery and Reinvestment Act aus dem Jahr 2009 habe dazu geführt, dass die Arbeitslosigkeit Ende 2010 niedriger gewesen wäre als ohne das Paket. Immer noch 56\% der Befragten äußerten, dass unter Berücksichtigung aller Kosten und Nutzen das Paket positiv zu bewerten sei (IGM Forum 2014). Für Deutschland hat eine Umfrage unter den Mitglieder des Vereins für Socialpolitik ergeben, dass der Anteil jener, die der Aussage „Finanzpolitik kann ein effektives Instrument sein, den Konjunkturzyklus zu stabilisieren“ ohne Einschränkungen zustimmen, von 2006 bis 2015 von 12,1 auf 36\% gestiegen ist, und der Anteil jener, die diese Aussage kategorisch ablehnen, von knapp 30\% auf 8,4\% gefallen ist (Fricke 2017). ${ }^{2}$

Zur Wirkung von Austerität hat zumindest der IWF ein weit beachtetes Papier veröffentlicht, dass die Fiskalmultiplikatoren vor der Krise unterschätzt worden seien (Blanchard und Leigh 2013); bei den Arbeiten von Reinhard und Rogoff (2010) zu nicht-keynesianischen Effekten hat sich herausgestellt, dass der zuvor proklamier-

\footnotetext{
${ }^{2}$ In beiden Umfragen gaben noch einmal rund $50 \%$ an, dem Statement „,mit Einschränkungen“ zuzustimmen.
} 
te Schwellenwert von Staatsschulden von $90 \%$, ab der das Wirtschaftswachstum massiv nachlässt, auf einem Spreadsheet-Fehler beruhte.

Wie auch immer nun tatsächlich die Mehrheitsmeinung zur Wirkung von Fiskalpolitik ist, dieses Beispiel unterstreicht eigentlich noch einmal die Gefahr monoparadigmatischer Politikberatung. Nehmen wir einmal an, Kooths ablehnende Position diskretionärer Fiskalpolitik auch in tiefen Krisen entspräche tatsächlich der Meinung des Mainstreams. Gleichzeitig ist objektiv festzustellen, dass die Zustimmung zu dieser Mainstream-Position unter Ökonomen sich massiv zugunsten einer alternativen Position der Wirksamkeit von diskretionärer Finanzpolitik verschoben hat. Kooths geforderte einheitliche (Mehrheits-)Empfehlung etwa des Sachverständigenrates würde hier bedeuten, diese Verschiebung in der Zustimmung unter Ökonomen der Politik in den wichtigen Gutachten nicht dargelegt würde und dass bei einer neuen Krise die (Mehrheits-)Empfehlung zu Konjunkturpaketen ähnlich ablehnend ausfallen müsste wie jene im Jahresgutachten 2008/9.

\section{Literatur}

Blanchard, O., \& Leigh, D. (2013). Growth Forecast Errors and Fiscal Multipliers. IMF Working Paper, Bd. EP/13/1. Washington, DC: IMF. https://www.imf.org/external/pubs/ft/wp/2013/wp1301.pdf

Fricke, T. (2017). Altes Einheitsdenken oder neue Vielfalt? Eine systematische Auswertung der großen Umfragen unter Deutschlands Wirtschaftswissenschaftler_innen. FGW Studie Neues Ökonomisches Denken, Bd. 3. Düsseldorf: FGW - Forschungsinstitut für gesellschaftliche Weiterentwicklung e.V.. http://www.fgw-nrw.de/fileadmin/user_upload/NOED-Studie-03-Fricke-A1-Web-komplett.pdf

Fuchs, V.R., Krueger, A.B., \& Poterba, J. M. (1998). Economists' views about parameters, values, and policies. Survey results in labor and public economics. Journal of Economic Literature, 36(3), 1387-1425.

Gordon, R., \& Dahl, G. B. (2013). Views among economists. Professional consensus or point-counterpoint? American Economic Review, 103(3), 629-635.

Krupp, H.-J. (2018). Zu den Grundlagen wissenschaftlicher Politikberatung. Wirtschaftsdienst, 98(5), 325-332. https://doi.org/10.1007/s10273-018-2294-7.

May, A. M., McGarvey, M.G., \& Kucera, D. (2018). Gender and European economic policy. A survey of the views of European economists on contemporary economic policy. Kyklos, 71(1), 162-183.

Reinhart, C.M., \& Rogoff, K.S. (2010). Growth in a time of debt. NBER Working Paper, Bd. 15639. Cambridge: The National Bureau of Ergonomic Research. https://www.nber.org/papers/w15639.pdf

Solomon, M. (2007). Social empiricism. Cambridge: MIT Press. 